\title{
Treatment of non-carious lesions: Diagnosis, restorative materials and techniques
}

Caroline Mathias ${ }^{1}$, Laura Nobre Ferraz' ${ }^{1}$, Débora Alves Nunes Leite Lima ${ }^{2}$, Giselle Maria Marchi ${ }^{2 \star}$

\begin{abstract}
1 PhD student, MSc, DDS, Department of Restorative Dentistry, Piracicaba Dental School, University of Campinas, Piracicaba, SP, Brazil.

2 Professor, PhD, MSc, DDS, Department of Restorative Dentistry, Piracicaba Dental School, University of Campinas, Piracicaba, SP, Brazil
\end{abstract}

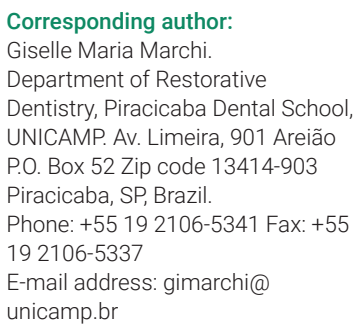

Received: March 04, 2018

Accepted: May 30, 2018
Management of non-carious cervical lesions (NCCLs) still is a challenge in clinical practice. The diagnosis is the key to treat these lesions successfully. Aim/Case report: Therefore, the aim of this case report is to describe the diagnosis and treatment of generalized abfraction lesions associated to abrasion lesions of a 43 years old patient and to discuss considerations about the technique and materials to be used appropriately. After the diagnosis, a treatment plan with preventive and restorative approach was elaborated. The occlusal adjustment was performed to distribute the contact points and the patient was instructed in relation to tooth brushing habits. It was also recommended that the patient use a dentifrice with potassium nitrate for the control of sensitivity. For lesions over $1 \mathrm{~mm}$ deep, the restorative treatment was performed using the self-etching adhesive system and nanohybrid composite by the stratification technique. Unsatisfactory restorations have been replaced. Occlusal splint was done and the patient was instructed to use the device every day overnight. One year after treatment, follow-up was performed. The appearance of new NCCLs was not observed. There was no progression of lesions smaller than $1 \mathrm{~mm}$. There was no loss of restorations. All restorations were fully satisfactory and the patient reported absence of dentin hypersensitivity. Conclusion: The treatment performed besides being aesthetically satisfactory after 1 year resulted in the control of the disease, preventing the appearance of new lesions and guaranteeing a better quality of life to the patient.

Keywords: Tooth wear. Diagnosis. Dental restoration. 


\section{Introduction}

The term non-carious cervical lesions (NCCLs) refers to the loss of dental tissue caused by processes that do not involve bacteria'. Its etiology is considered multifactorial and complex involving processes such as biocorrosion, abrasion and abfraction?2.

Dental biocorrosion is characterized by chemical dissolution of mineralized dental tissues by acids of non-bacterial origin ${ }^{3}$. Abrasion, occurs by objects or substances that are in frequent contact with dental surfaces, such as toothbrushing and abrasive dentifrices, resulting in mechanical wear ${ }^{4,5}$. The abfraction theory is based mainly on the concentration of tensions in the cervical areas, which cause flexion of the tooth resulting in microfractures and loss of dental ${ }^{6,7}$. Considering that occlusal forces may cause stress concentration, occlusal interferences, premature contact, bruxism and clenching could contribute to the etiology of NCCLs ${ }^{8}$. However, some studies have proposed a combination of occlusal tensions with abrasion and biocorrosion in the development of lesions, leading to a conclusion that the progression of abfraction may be multifactorial9,10.

Clinical appearance of NCCLs can vary from soft depressions to large wedges or discshaped lesions, characterized by uniform loss of hard tissue, predominantly on the buccal surfaces, independently of the affected teeth, near the cement-enamel junction (CEJ) ${ }^{11}$. These lesions may lead to biofilm growth and are often associated with irritation and gingival recession ${ }^{12}$, causing structural fragilities reflected in a poor rootcrown relationship and aesthetic complaints ${ }^{13}$. Also, NCCLs are a predisposing factor for dentin hypersensitivity $(\mathrm{DH})^{14}$. $\mathrm{DH}$ is a painful condition that occurs when the dentin is exposed to the oral environment. DH is certainly a reflection of the cumulative exposure to causal agents, which is strongly related to age, with a higher incidence in the age group of 31-50 years, and, in older age groups, $\mathrm{DH}$ tends to decrease due to the repair processes of the pulp ${ }^{14}$.

Successful prevention and management of abfraction or any NCCLs requires an understanding of the risk factors and how these factors change over time in individual patients $\mathrm{s}^{15}$. Preventive interventions may include counseling for changes in patient's behavior, such as diet, brushing technique, use of protective night guards to reduce clenching or bruxism, use of chewing gums to increase salivary flow, and/or to seek therapy or medical attention if there is a potential, intrinsic medical or mental condition ${ }^{15}$. Other treatment options include the following: monitoring of lesion progression, occlusal adjustments, occlusal splints, techniques to alleviate hypersensitity, placement of restorations, and root coverage surgical procedures in combination with restorations ${ }^{15}$. Since NCCLs often involve the simultaneous loss of tooth structure and gingival recession the diagnostic assessment and treatment should be based on a multidisciplinary approach to periodontal and restorative aspects ${ }^{13}$. As treatment strategies may vary according to the type of gingival recession, marginal level and extent of NCCL, the clinical characteristics of each defect should be considered prior to treatment ${ }^{13}$.

The NCCLs restorative treatment improves oral hygiene maintenance by the patient. It also helps to decrease thermal sensitivity, improving aesthetics and strengthening teeth $^{16}$. The most important criterion for restoration is retention and restoring these lesions represents a challenge in dentistry ${ }^{16,17}$. In most cases, lesions involve margins 
on enamel and dentin and also due to the increased amount of scleral dentin, NCCLs present low permeability and hypermineralization, resulting in a surface that is not favorable for adhesion 15,16 . Along with restoration, a variety of treatment strategies have also been proposed as occlusal adjustments, occlusal plaques and elimination of parafunctional habits ${ }^{18}$. Therefore, the aim of the present case report is to describe and discuss the current understanding of treatment for NCCLs with a preventive and restorative approach, and to describe the techniques and materials indicated for such treatments.

\section{Case Report}

A 43 year old female presented to the clinic of Piracicaba Dental School, State University of Campinas - São Paulo, with aesthetic complaint of teeth and dental hypersensitivity to thermal (cold) and air stimulus. For the diagnosis and treatment planning, full-mouth radiographs, a periodontal examination, and the fabrication of study casts were performed, and a full-mouth photograph was taken.

The patient present NCCLs on teeth 11 and 21 on the buccal surface, at a lower depth, polished and with no defined contour (Figure 1). Also presented NCCLs with dentin exposure, wedge-shaped or V-shaped with clearly defined internal and external angles located in the cervical region of the buccal surface of the teeth $13,16,24,26,34,35$, 36, 44, 45, 46, 47 (Figure 2a and 2b). Furthermore, the teeth 12, 14, 15, 23, 25 and 27 had unsatisfactory Class $V$ restorations due to bad adaptation at the margins of the underlying lesion (Figure 2a and 2b). In addition, all teeth had a highly polished buccal surface. Multiple gingival recessions were present in all teeth that presented cervical lesion. The patient reported the use of a rigid dental brush and the use of excessive force during brushing. Also, occlusal interferences, premature contact and the presence of unsatisfactory occlusal restorations on teeth $36,45,46$ and 47 were observed (Figure $2 \mathrm{c}$ and $2 \mathrm{~d}$ ). The patient reported clenching in situations of stress and especially during the night.

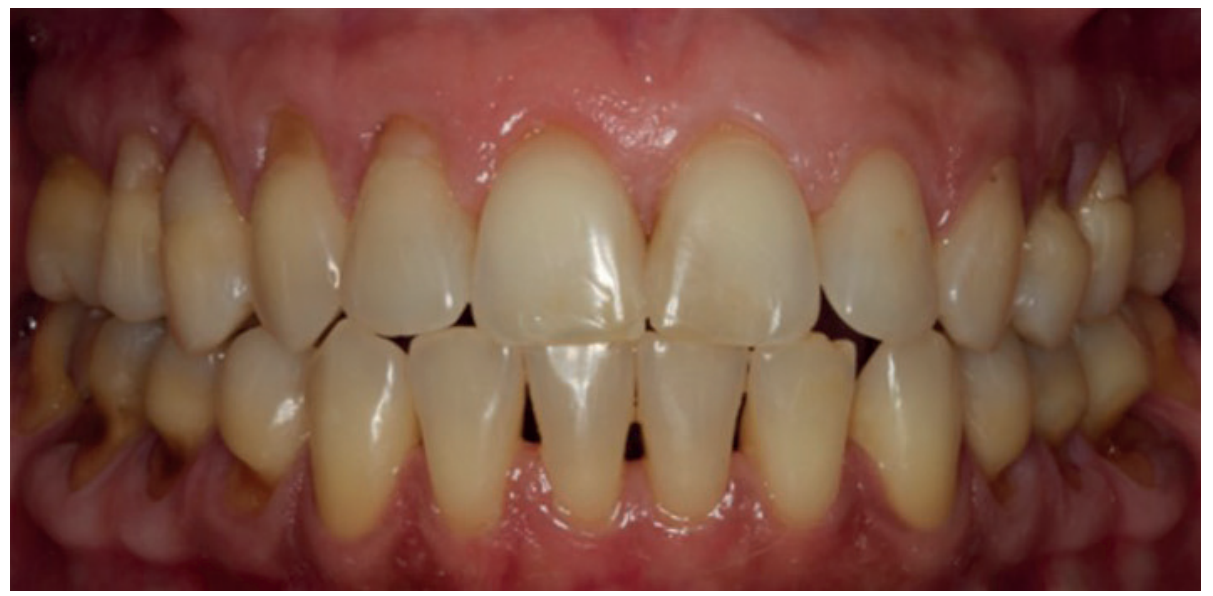

Figure 1. Preoperative frontal view. Note the presence of a non-carious cervical lesion on the maxillary central incisor, the right lateral incisor, and the left premolar. Old resin restorations on right canines, left and right premolars showed ill-fitting margins. 

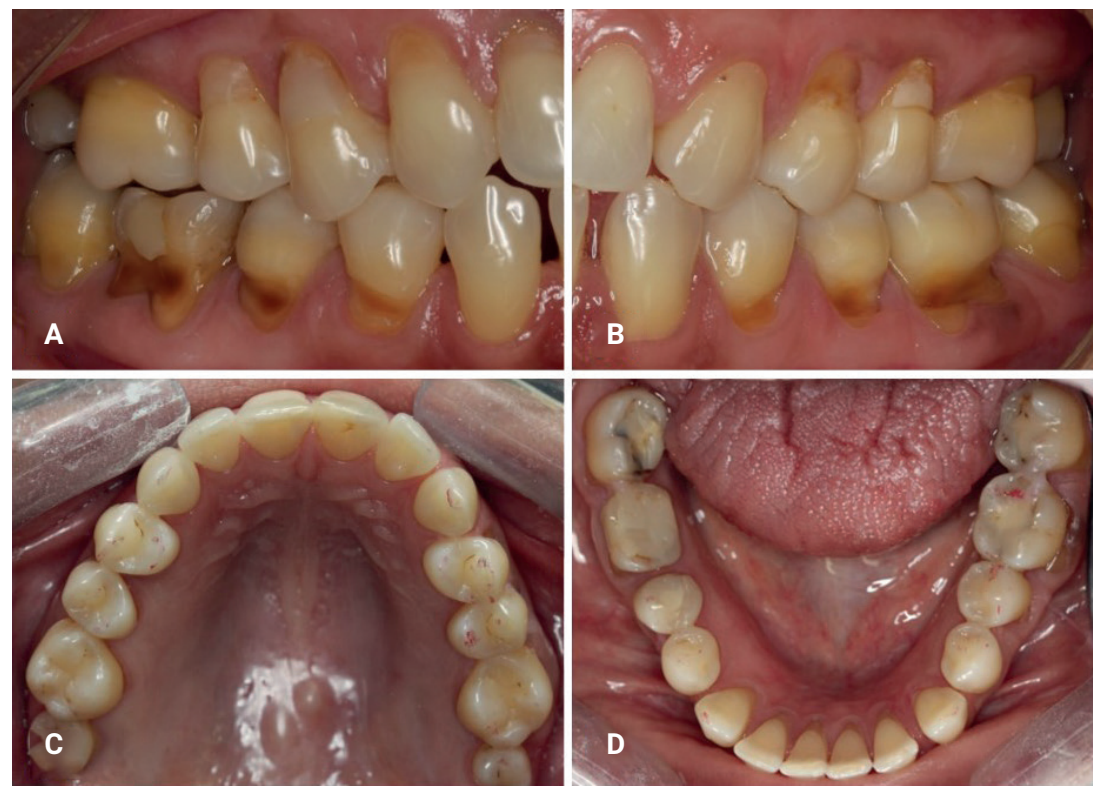

Figure 2. Initial clinical appearance of the posterior teeth. (a) NCCLs in the lower molars and premolars, superior canine and unsatisfactory restorations in the upper molar teeth. (b) NCCLs in the molars and premolars and unsatisfactory restorations in the upper second molar. (c) View of the occlusal contact points of maxillary teeth with excessive points of contact on the left side. (d) view of the occlusal contact points of the mandibular teeth with excessive points of contact on the posterior teeth on the left side.

The operative dentistry team diagnosed chronic sensitivity due to loss of enamel tissue and dentin exposure. The etiology of cervical lesions of the posterior teeth was considered multifactorial due to the association of abfraction and abrasion factors and characteristics. The cervical lesions of the anterior teeth were probably caused only by the abrasion process. After the diagnosis, a treatment plan with preventive and restorative approach was elaborated.

\section{Preventive intervention and control of dental sensitivity}

Preventive intervention was performed to aim controlling the etiological agent avoiding the appearance of new lesions and the progression of existing lesions. Thus, the patient was instructed to perform brushing with vertical movements, using little force and avoiding brushing for very long periods. For the treatment of sensitivity was indicated brushing 3 times daily using potassium nitrate dentifrice (Sensodyne Extra Fresh, SmithKline Beecham Consumer Heathcare, Berkshire, United Kingdom). To improve occlusal harmony and distribution of contact points, occlusal adjustment was performed by reduce heavy contacts and removing of premature contacts.

\section{Restorative treatment}

For the NCCLs of the teeth 11 and 21 only the monitoring of the lesion at regular intervals is sufficient because they present less than $1 \mathrm{~mm}$ in depth. For the teeth 13, 16, 24, $26,34,35,36,44,45,46,47$ the restorative treatment for rehabilitation of aesthetics and 
health due to structural loss was indicated. For this case report, the nanohybrid composite Empress Direct (Ivoclar-Vivadent, Liechtenstein) was chosen. Firstly, dental prophylaxis was performed using a mixture of stone powders and water, for the removal of the biofilm. After that, the choice of color was made by photopolymerization of small increments of composite resin on the vestibular surface of the tooth, close to the region to be restored and the color A3.5 was chosen for the dentin and A3 for enamel. Relative isolation of operative field was performed. A retracting wire was inserted into the gingival sulcus to expose the margin of the lesion and also to control the inflow of the crevicular fluid (Figure 3a). Also, were used high-power suckers, cotton rollers and front lip retractor. A self-etching adhesive system (Clearfil SE Bond, Kurary, Tokyo, Japan) was used. For this, just the enamel was conditioned with $35 \%$ phosphoric acid (Ultra-Etch, Ultradent Products Inc.) for 30 seconds (Figure 3b). The tooth was then washed with a water spray for $30 \mathrm{sec}-$ onds and dried with cotton ball. The primer of the adhesive system was actively applied for the time of 20 seconds (Figure 3c) followed by slight air drying and the application of two bond layers (Figure $3 \mathrm{~d}$ ). The photopolymerization was performed for 40 seconds using a LED light device (Radii Plus, SDI, Melbourne, Victoria, Australia).
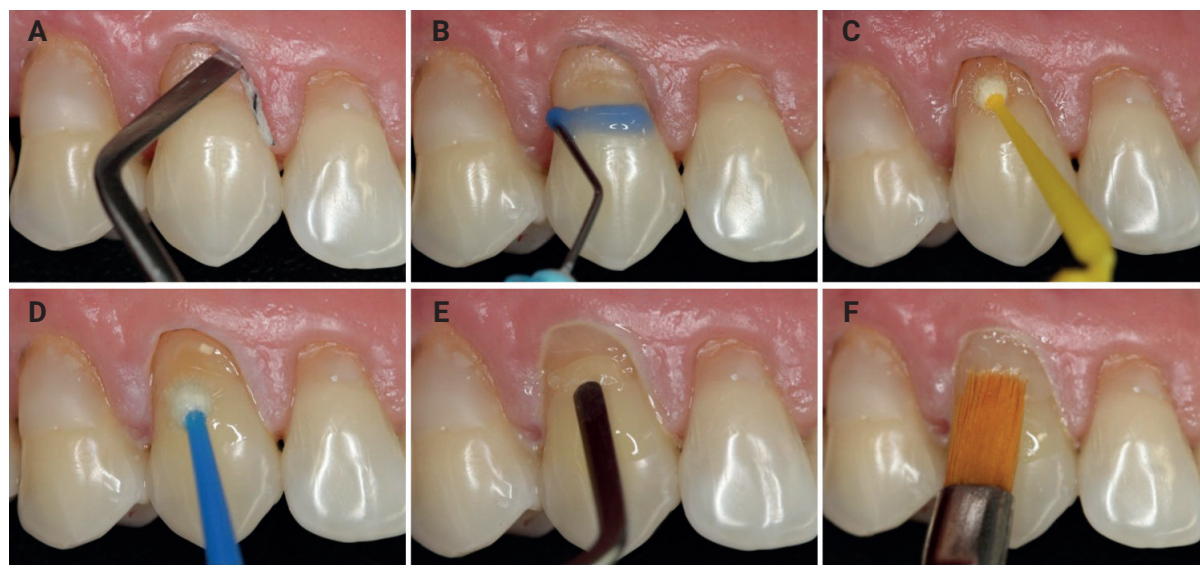

Figure 3. Restorative procedures on the righ maxillary canine. (a) Retracting wire insertion. (b) Selective phosphoric acid etching of the enamel. (c) Self-etching adhesive system application (primer). (d) Selfetching adhesive system application (bond). (e) Resin increase for dentin. (f) Resin increase for enamel.

In order to reduce the tension generated by the polymerization, the stratification technique was performed using small increments of composite resin with dentin characteristics, first the cervical margin and then the occlusal margin of the cavity (Figure 3e). After the dentin reconstruction, a layer of composite with enamel characteristics was applied to the cavity with the aid of a brush to reduce the excesses and obtain a smooth and uniform surface (Figure 3f). After insertion, each increment was photopolymerized for 20 seconds, according to the manufacturer's recommendation. After the final polymerization, the retraction wire was removed and the finishing procedures started.

Adhesive and resin excesses on the restoration margins were detected with an exploratory probe and removed with No. 12 scalpel blades and 2200F and 2200FF diamond 
tips. The diamond tip 3082 was also used to remove superficial excesses. After 48 hours, the finishing and polishing procedures were performed, using flexible disks with decreasing granulation (Figure $4 a, 4 b, 4 c$ and $4 d$ ) and abrasive rubbers (Figure $5 a$, $5 \mathrm{~b}$ and $5 \mathrm{c}$ ) until obtaining a highly smooth surface. For the polishing, silicon carbide brushes (Figure 5d) and polishing paste were used on felt disks (Figure 5e). In Figure $5 f$ the final result of such procedures are shown, where was obtained a perfectly adapted, smooth, shiny and polished restoration, which mimics the natural tooth.
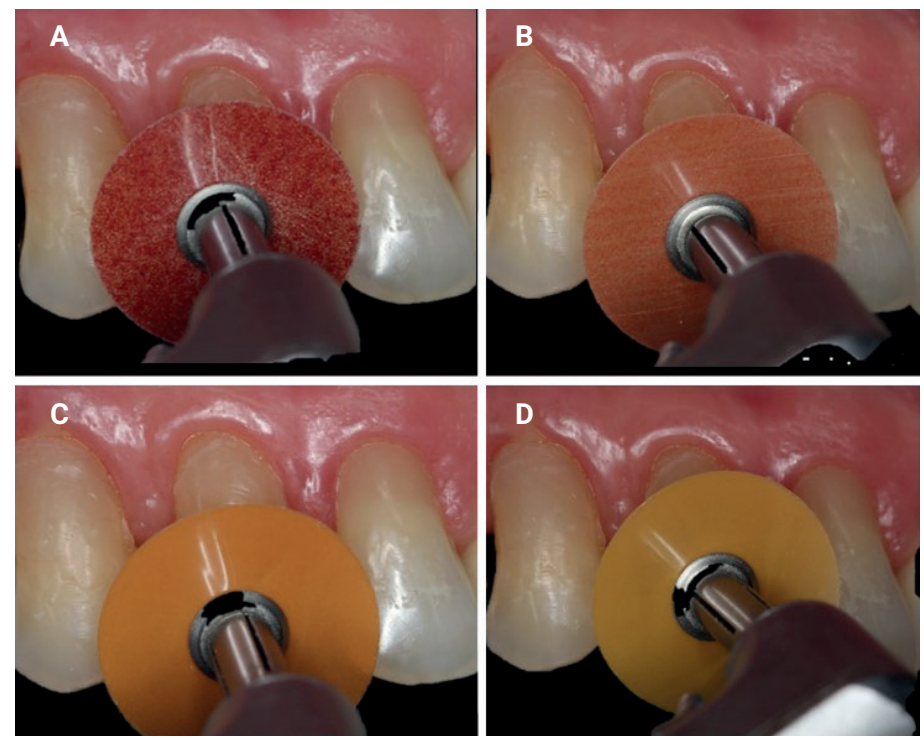

Figure 4. Finishing procedure of restoration. (a, b, c and d) Use of flexible disks with decreasing granulation.
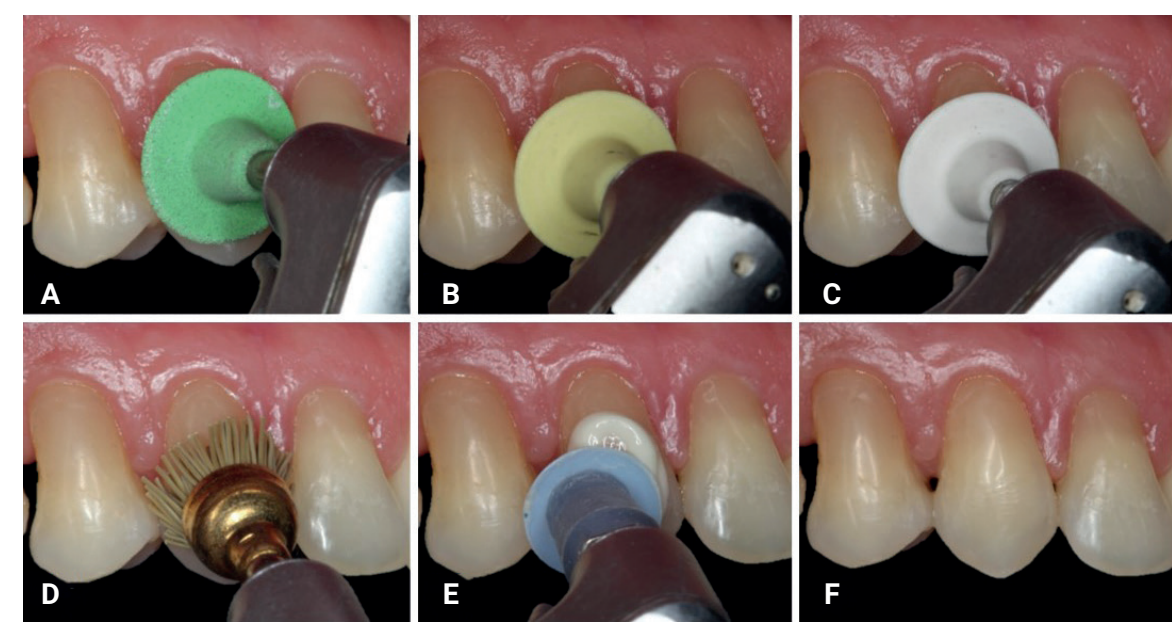

Figure 5. Finishing and polishing procedures. (a, b and c) Finishing with abrasive rubbers. (d) polishing with silicon carbide brush. (e) polishing with paste on felt disk. (f) Final aspect of restoration. 
After restoration of NCCLs, Class $\vee$ restorations were replaced for teeth 12, 14, 15, 23, 25 and 27. Class I restorations of the teeth 36 and 47 and the Class II restoration of the tooth 45 were also changed. The tooth 46 presented a great loss of dental tissue, thus indicating the placement of a total crown. Endodontic retreatment, fiberglass pin cementation and a monolithic zirconia crown were performed. Class III restoration of the tooth 11 and Class IV of the tooth 21 were replaced due to staining. At the end of all restorative procedures, occlusal adjustment was again performed (Figure 6a and 6b).

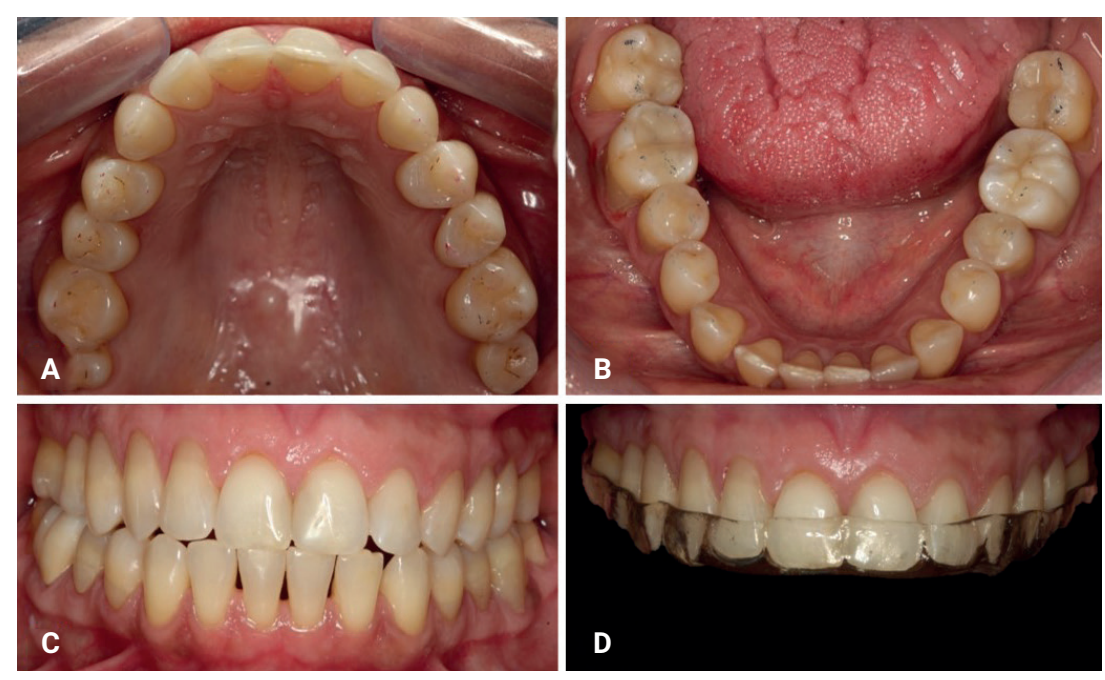

Figure 6. Final aspect of the restorations. (a) Occlusal view of maxillary teeth after termination of treatment showing well-distributed occlusal contact points (b) Occlusal view of mandibular teeth after treatment showing well-distributed occlusal contact points (c) Frontal view after treatment (d) Installation of the occlusal splints.

\section{Occlusal splints}

After the end of all the restorations occlusal splint was done and the patient was instructed to use the device every day overnight (Figure 6d).

\section{Follow-up}

One year after the end of restorations, the patient was contacted for follow-up.

When questioned about oral health the patient reported that the dental treatment performed resulted in a better quality of life mainly due to the absence of hypersensitivity to thermal (cold) and air stimulus. The patient changed her brushing habits, performed the oral hygiene according to instruction and used the occlusal splint every day during the night.

During the clinical examination, the presence of new cervical lesions was not detected. It was observed that there was no progression of the lesions smaller than $1 \mathrm{~mm}$ of the teeth 11 and 21 . All restorative procedures performed were aesthetically satisfactory. 
There were no losses of the restorations. All class $V$ restorations were fully satisfactory with perfectly matched edges and good surface polishing. Class I restorations of teeth 36 and 47 and tooth II class II also remained satisfactory without any maladjustment, fracture or excessive wear of the restorative material. The total crown of the tooth 46 also remained intact (Figure 7).
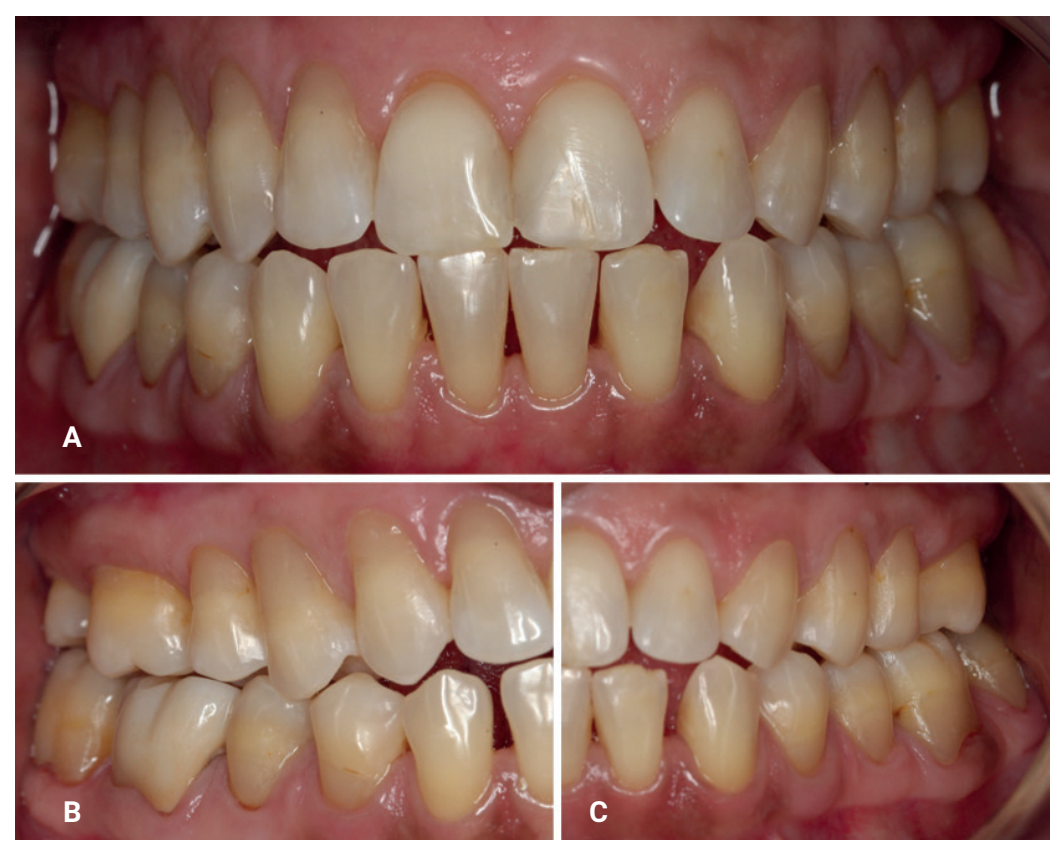

Figure 7. Aspects of the restorations after 1-year follow up. (a) Frontal view of restoration in function after 1-year placement. (b) Left view of restorations. The crown and Class V restorations well adapted. (c) Right view of restorations. Class $\mathrm{V}$ restorations without margin cracks and presenting good adaptation.

\section{Discussion}

The NCCLs etiology is still controversial, abfraction lesions are believed to be caused by tooth flexure arising from cyclic and eccentric occlusal forces, besides parafunction ${ }^{8,19}$. Other modifying factors must be considered in the abfraction etiology as the composition, buffering capacity, flow rate, viscosity and $\mathrm{pH}$ of saliva ${ }^{8}$. These lesions are considered multifactorial, once according to traditional theory of abfraction etiology, the enamel micro cracks, formed by tensile stresses under non-axial loading, can predisposed the tooth tissue to biocorrosion and abrasion ${ }^{8}$. However, the lack of clinical studies and the fact that NCCLs are often found in buccal faces of teeth, it reinforces the belief of some authors that the cause-effect relationship between the abfraction lesions and occlusal loading can not be confirmed $15,16,20$. Therefore, the clinicians often have difficulties in correctly diagnosing the lesions, which require a careful anamnesis to treat according to their etiology.

The first step on treatment of NCCLs lesions is to stop or prevent further progression of the lesions by controlling all potential etiologic factors that can be associated to 
these lesions ${ }^{21}$. For this, the patient should be instructed to avoid the consumption of acid fruits and to perform brushing with vertical movements, using little force during short periods. Vertical brushing promotes three times less wear and tear than horizontal brushing ${ }^{22}$. Also, a proper occlusal adjustment should be performed to eliminate premature contacts and promote a balanced occlusion, often requiring the replacement of deficient occlusal restorations that do not allow adequate contact with the opposing tooth ${ }^{23}$. When the treatment is not based on the etiological agent, even though the lesion is restored, probably it can occurs the progression of the underlying lesion and consequently misalignment of the composite resin at the margins of the lesion, leading it to failure ${ }^{15}$.

$\mathrm{DH}$ is associated to ageing, presence of NCCLs, premature contacts and frequent consumption of erosive fruit juices ${ }^{24}$. The treatment of DH still lacks a standard proto$\mathrm{col}$ to solve the patient discomfort. Because of this, recurrences are common ${ }^{25}$. Freitas et al. ${ }^{25}$ reported that is more efficient to restore a tooth with moderate or severe $\mathrm{DH}$ as soon as possible instead of trying to desensitize it before the filling procedure. Besides, the choice of the desensitizing agent is important if the tooth will posteriorly be restored, because agents that contain sodium and calcium fluoride may reduce the bond strength of adhesives ${ }^{26}$. Therefore, no desensitizing agent was recommended for the patient in this study.

There are no consensus or guidelines in the literature about when abfraction lesions should be restored ${ }^{15}$. However, when these lesions are less than $1 \mathrm{~mm}$ in depth, according to Shetty et al. ${ }^{27}$ no restorative procedure is necessary, only monitoring at regular intervals. Because of this, the NCCLs of the patient's teeth 11 and 21 were not restored. The advantages in restoring the NCCLs are: better maintenance of oral hygiene by the patiente, decreasing of thermal sensitivity, improvement of esthetics and strengthening of the teeth ${ }^{27}$.

One of the challenges in restoring these lesions is the difficulty with moisture control ${ }^{15}$. Relative isolation of operative field was preferred in this case report, because it was necessary to completely visualize the dentogengival regions, which would not be possible with the absolute isolation. In addition, the teeth to be restored presented lesions at the gingival level and did not present hard tissue for the retention of the staples.

Clinical studies have shown that NCCLs restorations have higher rate of failure in the cervical area, due to constant deformation of the tooth caused by parafunctional habits of the patient ${ }^{28}$. Also, NCCLs lesions have large amount of sclerotic and hypermineralized dentin that affect it permeability for adhesives agents ${ }^{15,16}$. Therefore, another important criterion for these kind of restoration is the retention ${ }^{16}$. While some studies have shown no difference between retention rates of etch-and-rinse and self-etch adhesives after short and long-term clinica|29,30, others have shown that self-etch adhesives should be preferred as long as the enamel is conditioned with phosphoric acid ${ }^{15,28}$.

Restorative materials with low modulus of elasticity should be the first choice to restore abfraction lesions ${ }^{15,16,31}$. When the aesthetic is not a concern, glass ionomer should be used, because these restorations revealed the best results in the context of clinical effectiveness ${ }^{15,27}$. However, methacrylate-based composites are usually the first choice to restore these lesions, being the gold-standard, due to their mechanical 
properties and better esthetic compared to glass ionomers ${ }^{32}$. Microfilled composites demonstrate a greater elasticity than hybrid composites, even presenting excellent polishing properties ${ }^{16}$. Because of this, a nanohybrid composite was selected to restore the patient's lesions, due to its excellent mechanical properties and good polishing. The polishing procedure is important to decrease the biofilm adhesion on these restored surfaces, avoiding inflammation and gingival recession ${ }^{13}$.

Because of the association between occlusal stress and abfraction lesions, occlusal splints have been proposed as an alternative and conservative treatment for the management of abfraction lesions, to reduce the amount of nocturnal bruxism or clenching and to preserve the restorations ${ }^{33}$. Besides, the use of occlusal splints can avoid other damages for dental, periodontal and musculoskeletal tissues, caused by nocturnal parafunctional activity ${ }^{33}$.

To achieve the success of NCCL lesions treatment it is necessary to identify correctly the etiology of the lesion, to act on them and to carefully select the restorative materials to be used. Also, it is important to consider the patient's oral hygiene and follow-up should be performed for long-term maintenance of restorations.

The follow-up session was performed 1 year after treatment. It is advisable to monitor the progression of these lesions at regular intervals without any treatment intervention ${ }^{15}$. The assessment of lesion activity can be performed every 6 months to 12 months and during regular hygiene visits ${ }^{15}$. There was no progression of lesions smaller than $1 \mathrm{~mm}$ and no new lesions appeared. The orientation of brushing habits, occlusal adjustment and occlusal splints were determinant for this.

There were no losses of any of the class $\vee$ restorations performed. Cervical lesions do not provide micromechanical retention and an ineffective adhesion may result in the loss of the restoration. This type of substrate is a single substrate probably not found in any other region of the mouth ${ }^{34}$. Lesions are often characterized by the presence of physiologically and pathologically altered scleral dentin, resulting in partial or complete obliteration of the dentinal tubules. These lesions present a complex structure with high variability of tubule occlusion ${ }^{34}$. Because of such characteristics, bond strengths to NCCL have consistently been reported to be $20 \%-50 \%$ lower than bonds made to sound dentin ${ }^{34}$. Nevertheless, in this clinical case the restorative protocol used resulted in a satisfactory performance after 1 year with perfectly adapted restorations and with good surface polishing, due to the focus on NCCLs etiology and adequate restoration technique performed. In addition, the restorative procedure resulted in the absence of $\mathrm{DH}$, since restorative treatments of NCCLs help to decrease the thermal sensitivity ${ }^{16}$.

NCCLs still represent a challenge for clinical practice and the accurate diagnosis and the choice of proper treatment is the key of success for theses lesions. The restorative treatment must be considered for dentin hypersensibility and for the re-establishing of dental esthetics. However, a good finishing and polishing is essential for gingival health. Overall, the treatment of NCCLs should be based on its etiology.

\section{Conflict of Interest}

No potential conflict of interest relevant to this article was reported. 


\section{References}

1. Walter C, Kress E, Götz H, Taylor K, Willershausen I, Zampelis A. The anatomy of non-carious cervical lesions. Clin Oral Investig. 2014 Jan;18(1):139-46. doi: 10.1007/s00784-013-0960-0.

2. Szesz A, Perreiras S, Martini E, Reis A, Loguercio A. Effect of flowable composites on the clinical performance of non-carious cervical lesions: a systematic review and meta-analysis. J Dent. 2017 Oct;65:11-21. doi: 10.1016/j.jdent.2017.07.007.

3. Maggio B, Guibert RG, Mason SC, Karwal R, Rees GD, Kelly S, et al. Evaluation of mouthrinse and dentifrice regimens in an in situ erosion remineralisation model. J Dent. 2010 Nov;38 Suppl 3:S37-44. doi: 10.1016/S0300-5712(11)70007-0

4. Litonjua L, Andreana S, Cohen R. Toothbrush abrasions and noncarious cervical lesions: evolving concepts. Compend Contin Educ Dent. 2005 Nov;26(11):767-76.

5. Shellis R, Addy M. The interactions between attrition, abrasion and erosion in tooth wear. Monogr Oral Sci. 2014;25:32-45. doi: 10.1159/000359936.

6. Vasudeva G, Bogra P. The effect of occlusal restoration and loading on the development of abfraction lesions: a finite element study. J Conserv Dent. 2008 Jul;11(3):117-20. doi: 10.4103/0972-0707.45250.

7. Grippo JO, Simring M, Schreiner S. Attrition, abrasion, corrosion and abfraction revisited. J Am Dent Assoc. 2004 Aug;135(8):1109-18; quiz 1163-5.

8. Grippo JO, Simring M, Coleman TA. Abfraction, abrasion, biocorrosion, and the enigma of noncarious cervical lesions: A 20-year perspective. J Esthet Restor Dent. 2012 Feb;24(1):10-23. doi: 10.1111/j.1708-8240.2011.00487.x

9. Spranger $\mathrm{H}$. Investigation into the genesis of angular lesions at the cervical region of teeth. Quintessence Int. 1995 Feb;26(2):149-54.

10. Lee WC, Eakle WS. Stress-induced cervical lesions: review of advances in the past 10 years. $J$ Prosthet Dent. 1996 May;75(5):487-94.

11. Bartlett DW, Shah P. A critical review of non-carious cervical (wear) lesions and the role of abfraction, erosion, and abrasion. J Dent Res. 2006 Apr;85(4):306-12.

12. Aw TC, Lepe $X$, Johnson GH, Mancl L. Characteristics of noncarious cervical lesions: a clinical investigation. J Am Dent Assoc. 2002 Jun;133(6):725-33.

13. Yang $\mathrm{S}$, Lee $\mathrm{H}$. A combined approach to non-carious cervical lesions associated with gingival recession. Restor Dent Endod. 2016 Aug;41(3):218-24. doi: 10.5395/rde.2016.41.3.218.

14. Moraschini V, Costa LS, Santos GO. Effectiveness for dentin hypersensitivity treatment of noncarious cervical lesions: a meta-analysis. Clin Oral Investig. 2018 Mar;22(2):617-631. doi: 10.1007/ s00784-017-2330-9.

15. Nascimento MM, Dilbone DA, Pereira PN, Duarte WR, Geraldeli S, Delgado AJ. Abfraction lesions: etiology, diagnosis, and treatment options. Clin Cosmet Investig Dent. 2016 May 3;8:79-87. doi: 10.2147/CCIDE.S63465.

16. Sarode G, Sarode S. Abfraction: a review. J Oral Maxillofac Pathol. 2013 May; 17(2):222-7. doi: 10.4103/0973-029X.119788.

17. Heymann HO. Examining tooth flexure effects on cervical restorations: a two-year clinical study. J Am Dent Assoc. 1991 May;122(5):41-7.

18. Estafan A, Bartlett D, Goldstein G. A survey of management strategies for noncarious cervical lesions. Int J Prosthodont. 2014 Jan-Feb;27(1):87-90. doi: 10.11607/ijp.3585.

19. Antonelli JR, Hottel TL, Garcia-godoy F. Abfraction lesions--where do they come from? A review of the literature. J Tenn Dent Assoc. 2013 Spring-Summer;93(1):14-21. 
20. Leal NMS, Silva JL, Benigno MIM, Bemerguy EA, Meira JBC, Ballester RY. How mechanical stresses modulate enamel demineralization in non-carious cervical lesions? J Mech Behav Biomed Mater. 2017 Feb;66:50-57. doi: 10.1016/j.jmbbm.2016.11.003.

21. Pereira AG, Teixeira DNR, Soares MPCM, Gonzaga RCQ, Fernandes-Neto AJ, Soares PV. Periodontal and Restorative Treatment of gingival recession associated with non-carious cervical lesions: case study. J Int Acad Periodontol. 2016 Jan 14;18(1):16-22.

22. Jaeggi T, Lussi A. Toothbrush abrasion of erosively altered enamel after intraoral exposure to saliva: an in situ study. Caries Res. 1999 Nov-Dec;33(6):455-61.

23. Ichim IP, Schmidlin PR, Li Q, Kieser JA, Swain MV. Restoration of non-carious cervical lesions. Part II. Restorative material selection to minimise fracture. Dent Mater. 2007 Dec;23(12):1562-9.

24. Yoshizaki KT, Francisconi-dos-Rios LF, Sobral MAP, Aranha ACC, Mendes FM, Scaramucci T. Clinical features and factors associated with non-carious cervical lesions and dentin hypersensitivity. J Oral Rehabil. 2017 Feb;44(2):112-118. doi: 10.1111/joor.12469.

25. Freitas S da S, Sousa LLA, Moita Neto JM, Mendes RF, Prado Junior RR. Dentin hypersensitivity treatment of non-carious cervical lesions - a single-blind, split-mouth study. Braz Oral Res. 2015;29:45. doi: 10.1590/1807-3107BOR-2015.vol29.0045.

26. Külünk Ş, Saraç D, Külünk T, Karakaş Ö. The effects of different desensitizing agents on the shear bond strength of adhesive resin cement to dentin. J Esthet Restor Dent. 2011 Dec;23(6):380-7. doi: 10.1111/j.1708-8240.2011.00415.x

27. Shetty SM, Shetty RG, Mattigatti S, Managoli NA, Rairam SG, Patil AM. No carious cervical lesions: abfraction. J Int Oral Health. 2013 Oct;5(5):143-6.

28. Tuncer D, Yazici AR, Özgünaltay G, Dayangac B. Clinical evaluation of different adhesives used in the restoration of non-carious cervical lesions: 24-month results. Aust Dent J. 2013 Mar;58(1):94-100. doi: 10.1111/adj.12028.

29. Loguercio AD, Luque-Martinez IV, Fuentes S, Reis A, Muñoz MA. Effect of dentin roughness on the adhesive performance in non-carious cervical lesions: a double-blind randomized clinical trial. J Dent. 2018 Feb;69:60-69. doi: 10.1016/j.jdent.2017.09.011.

30. Van Dijken JW V. A prospective 8-year evaluation of a mild two-step self-etching adhesive and a heavily filled two-step etch-and-rinse system in non-carious cervical lesions. Dent Mater. 2010 Sep;26(9):940-6. doi: 10.1016/j.dental.2010.05.009.

31. Srirekha A, Bashetty K. A comparative analysis of restorative materials used in abfraction lesions in tooth with and without occlusal restoration: Three-dimensional finite element analysis. J Conserv Dent. 2013 Mar;16(2):157-61. doi: 10.4103/0972-0707.108200.

32. Cieplik F, Scholz KJ, Tabenski I, May S, Hiller KA, Schmalz G, et al. Flowable composites for restoration of non-carious cervical lesions: results after five years. Dent Mater. 2017 Dec;33(12):e428-e437. doi: 10.1016/j.dental.2017.09.012.

33. Tsiggos N, Tortopidis D, Hatzikyriakos A, Menexes G. Association between self-reported bruxism activity and occurrence of dental attrition, abfraction, and occlusal pits on natural teeth. J Prosthet Dent. 2008 Jul;100(1):41-6. doi: 10.1016/S0022-3913(08)60135-3.

34. Carvalho RM, Manso AP, Geraldeli S, Tay FR, Pashley DH. Durability of bonds and clinical success of adhesive restorations. Dent Mater. 2012 Jan;28(1):72-86. doi: 10.1016/j.dental.2011.09.011. 